\title{
Curvas de Lactação em Rebanhos da Raça Holandesa no Estado de Minas Gerais. Escolha do Modelo de Melhor Ajuste ${ }^{1}$
}

\section{Tarcísio de Moraes Gonçalves ${ }^{2}$, Antonio Ilson Gomes de Oliveira ${ }^{3}$, Rilke Tadeu Fonseca de Freitas $^{4}$, Idalmo Garcia Pereira ${ }^{5}$}

\begin{abstract}
RESUMO - Para selecionar o modelo de curva de lactação de melhor ajuste aos dados de produção de leite no dia de controle de animais da raça Holandesa no Estado de Minas Gerais, utilizaram-se 157.932 controles de 17.150 lactações, de 1990 a 1996, do arquivo inicial com 305.506 controles de 56.422 lactações, do Serviço de Controle Leiteiro da ACGHMG. Ajustaram-se os dados para os fatores: região, nível de produção, ano-estação de parto, classes de ordem e idade ao parto (COPI), grupo genético, número de controles e interações, além dos efeitos linear e quadrático da duração da lactação. Para verificar o melhor ajuste aos modelos Quadrático logarítmico, Gamma Incompleta, Regressão Múltipla e Multifásico (Monofásico e difásico), foram usados menor desvio padrão residual, $\mathrm{R}^{2}$ ajustado ao número de parâmetros das equações, autocorrelação de primeira ordem e teste de Durbin-Watson. O uso de uma função difásica para predizer a produção de leite em programas de seleção é recomendado, sendo também possível estimar, com base nessa função, a produção de leite em 305 dias.
\end{abstract}

Palavras-chave: ajuste de modelos, gamma-incompleta, multifásica, projeção da lactação, quadrático logarítmico

\section{Lactation Curve in Holstein Herds of Minas Gerais State. Choice of Model with the Best Fit}

\begin{abstract}
In order to select the model of the lactation curve that has the best adjustment for milk production records of Holstein cows in the Minas Gerais State, a total of 157,932 test day of 17,150 lactations from 305,506 test day of 56,422 lactations, from 1990 to 1996, provided by the milk recording service of the ACGHMG (Holstein Breed Association of Minas Gerais), were used. The data were adjusted for: region, management level, year -season of calving, COPI (parity and age of calving), genetic group, number of tests, interactions and linear and quadratic effects of lactation period. In order to compare results obtained using quadratic logarithmic, incomplete gamma, multiple regression and multiphasic (monophasic and diphasic) functions methods. The following statistics were used: lowest standard error, adjusted $\mathrm{R}^{2}$, first order auto-correlation and Durbin-Watson. It was concluded that the diphasic function was the best for predict milk yield in selection programs being possible to estimate the 305-day milk yield with this function.
\end{abstract}

Key Words: adjustment of models, incomplete gamma, multiphasic, quadratic logarithmic, projection of lactation

\section{Introdução}

O Brasil possui um grande rebanho bovino leiteiro, principalmente no Estado de Minas Gerais. Entretanto, os níveis de produtividade estão bastante aquém do desejado. Deficiências de manejo associadas à baixa qualidade genética do rebanho contribuem diretamente para esse resultado. Dentro desse contexto, um dos objetivos dos pesquisadores e produtores de leite tem sido usar uma metodologia de seleção que leve a um aumento dessa produção.

Para tanto, torna-se fundamental estimar, a partir de registros parciais, a produção total de leite de um animal em lactação. Considerando-se essa estimativa, é possível selecioná-lo com base em parte da sua lactação ou de seus descendentes. O avanço dos procedimentos estatísticos e o desenvolvimento de novas técnicas computacionais tornaram possível a utilização de métodos mais refinados, os quais conduzem a uma estimação mais acurada.

O resultado dessa estimação pode, dessa forma, ser fundamental nas tomadas de decisão em programas de seleção, bem como nas decisões de manejo. A produção total de leite está correlacionada com as produções nos diferentes estádios da lactação. Utilizam-se freqüentemente, para obter uma estima-

\footnotetext{
${ }^{1}$ Projeto financiado parcialmente pelo CNPq.

2 Prof. Assistente do DZO da UFLA - Doutorando UNESP Botucatu - Bolsista CNPq. E.mail: tarcisio@ufla.br

3 Prof. Titular Aposentado da UFLA. E.mail: ailson@ufla.br

${ }^{4}$ Prof. Adjunto do DZO da UFLA - CP 37 - 37200-000 - Lavras - MG. E.mail: rilke@ufla.br

${ }^{5}$ Prof. Adjunto da UFBA. E.mail: idalmo@cruz.mma.com.br
} 
tiva da produção de leite e gordura de uma vaca leiteira, os dados de produção diária de um animal durante certo estádio de sua lactação.

Muitos autores utilizam uma expressão algébrica para descrever a curva de lactação, visando a prever a produção de leite total a partir de registros parciais, e também a produção em cada estádio da lactação. A representação gráfica da produção de leite em função do tempo é denominada curva de lactação (Yadav et al., 1977). Um dos primeiros trabalhos a propor um modelo para descrever a curva de lactação foi o de Brody et al. (1923), o qual, segundo Wood (1967),não proporciona um ajuste adequado, pois não considera o pico da lactação.

Novos modelos foram propostos por outros autores, dentre eles Wood (1967), que propôs a função gamma incompleta, que pode ser usada para um único ou grupos de animais. Cobby \& Le Du (1978) verificaram pela análise dos resíduos que esste modelo tendia a superestimar a produção no início e no final da lactação e subestimar no meio da lactação.

Mais recentemente, Ali \& Schaeffer (1987) estudaram um modelo de regressão múltipla, o qual leva em consideração parâmetros relacionados à produção em 305 dias, ao pico de produção e ao formato da curva.

No intuito de corrigir falhas da função gamma incompleta na representação da curva de lactação, Grossman \& Koops (1988) usaram a função multifásica, a qual é um somatório de funções logísticas que melhor descrevem a curva de lactação do que a função gamma incompleta. Trabalhos de De Boer et al. (1989), Sherchand et al. (1995) e Vargas et al. (2000) têm evidenciado que a função difásica é a que fornece estimativas da produção de leite com maior acurácia.

Pelo exposto, objetiva-se com este trabalho definir um modelo para a curva de lactação que melhor se ajuste aos dados do controle leiteiro de animais da raça Holandesa no Estado de Minas Gerais.

\section{Material e Métodos}

Os dados utilizados foram provenientes do Serviço de Controle Leiteiro da Associação dos Criadores de Gado Holandês de Minas Gerais (ACGHMG), referentes ao período de 1985 a 1995 . Do arquivo inicial com 305.506 controles de 56.422 lactações, foram utilizados 157.932 controles de 17.150 lactações, de 1990 a 1996. Com base na produção média das vacas que pariram até os 42 meses de idade $(4.897,41 \mathrm{~kg})$, foram definidos dois níveis de produção, abaixo e igual ou acima dessa média. Os registros parciais foram ajustados para dia-padrão de controle, utilizando a interpolação de Lagrange (três pontos).

Utilizando-se os controles leiteiros mensais, estimou-se a produção de lei-te em 12 estádios da lactação, com intervalo de 30 dias $(22,52,82, \ldots, 342$ dias de lactação). As produções foram estimadas por interpolação de LAGKANGE, considerando-se os três pontos mais próximos do estádio da lactação a ser estimado. A seguinte fórmula foi utilizada:

$$
\operatorname{Pn}(\mathrm{X})=\sum_{i=0}^{n} f_{i} L_{i}(X)
$$

em que: $f_{i}$ é o valor assumido pela função no ponto $X_{i}$, ou seja, $\mathrm{f}_{\mathrm{i}}=\mathrm{f}\left(\mathrm{x}_{\mathrm{i}}\right) ; \mathrm{L}_{\mathrm{i}}(\mathrm{X})$ é o polinômio de LAGRANGE.

$$
L_{i}(X)=\coprod_{\substack{j=0 \\ j \neq 0}}^{3} \frac{\left(X-X_{j}\right)}{\left(X_{i}-X_{j}\right)}
$$

Após a separação dos rebanhos em níveis de produção, baixo e alto, foi gerada a variável COPI (classe de ordem de parição e idade do animal ao parto), sendo essa definida como: COPI 11 (animais de primeira parição com idade inferior a 30 meses), COPI 12 (primeira parição e idade entre 30 e 42 meses), COPI 21(segunda parição e idade inferior a 42 meses), COPI 22 (segunda parição e idade entre 42 e 60 meses), COPI 31 (terceira ou mais parições e idade inferior a 60 meses), COPI 32 (terceira ou mais parições e idade entre 60 e 72 meses) e COPI 33 (terceira ou mais parições e idade superior a 72 meses).

Foram estudados os modelos: Quadráticologarítmico, função Gamma Incompleta, Regressão Múltipla e Multifásicos (Monofásico \& difásico). Para a escolha da curva que melhor se ajustou às lactações, consideraram-se os métodos: menor desvio-padrão residual (Brown et al., 1976), $\mathrm{R}^{2}$ ajustado ao número de parâmetros das equações (Malhotra et al., 1980), auto-correlação de resíduos de primeira ordem e teste de Durbin-Watson (GironPineda, 1987).

Foram avaliadas, para cada modelo, as funções das estimativas dos parâmetros, produção inicial (utilização do modelo, sendo $\mathrm{t}=0$ ), pico de produção $\left(\mathrm{a}_{\mathrm{i}}\left(b_{i} / c_{i}\right)^{2} e^{-b_{i}}\right)$, produção em 305 dias, tempo até o 
pico e duração da fase (essa para os modelos multifásicos).

Após a definição do modelo difásico, foram geradas as curvas para cada nível de produção e cada uma das classes de ordem de parição e idade (COPI).

\section{Resultados e Discussão}

As equações que melhor se ajustaram aos dados, conforme os modelos, foram:

Modelo gamma incompleto: $\mathrm{Y}=19,0879 *\left(\mathrm{t}^{0,101}\right)$

$* \exp (0,002752 * \mathrm{t})$

Modelo monofásico: $\mathrm{Y}=13138,39 * 0,00202 *$ $\left(1-\tanh ^{2}(0,0202 *(\mathrm{t}-(-94,4775)))\right)$

Modelo difásico: $\mathrm{Y}=605,6448 * 0,0094 *\left(1-\tanh ^{2}\right.$ $(0,0094 *(\mathrm{t}-34,3691)))+8094,7903 * 0,0024 *$ $\left(1-\tanh ^{2}(0,0024 *(\mathrm{t}-80,4179))\right)$

Modelo quadrático-logarítmico: $\mathrm{Y}=16,781+$ $(-0,0861 \mathrm{t})+\left(-0,00007 \mathrm{t}^{2}\right)+3,097(\ln \mathrm{t})$

Modelo de regressão múltipla: $Y=21,3563-8,079$ $*(\mathrm{t} / 305)+1,4652 *((\mathrm{t} / 305) 2)+5,5345 * \ln (305 / \mathrm{t})-$ $1,5754 *((\ln (305 / \mathrm{t})) 2)$.

em que t é o número de dias entre o parto e o controle leiteiro.

Os resultados obtidos para $\mathrm{R}^{2}$ ajustado, autocorrelação de resíduo de primeira ordem, desvio padrão residual e teste de Durbin-Watson (Tabela 1) evidenciam que os modelos estudados ajustaram-se bem aos controles mensais de produção de leite, ao passo que a função monofásica (equação 2) apresentou um valor de desvio padrão residual mais elevado que as demais. Pela Figura 1 observa-se que a regressão múltipla (equação 5) é a que apresenta distribuição de resíduos mais próxima de zero.

Entretanto, pela Tabela 2 e Figura 2, nota-se que a produção de leite no início da lactação é subestimada pelas funções quadrático-logarítmica, gama incompleta e a de regressão múltipla, assemelhando-se aos resultados de Cobby \& Le Du (1978), sendo, entretanto, superestimada pela função monofásica.

Tabela 1 - Produções observadas e previstas segundo o estádio da lactação e os diferentes modelos

Table 1 - Actual and predicted mean test-day milk yields according the stage of lactation and the different models

\begin{tabular}{|c|c|c|c|c|c|c|c|}
\hline \multirow{2}{*}{$\begin{array}{c}\text { Estádio da } \\
\text { lactação } \\
\text { Stage of } \\
\text { lactation } \\
\text { Dias } \\
\text { Days }\end{array}$} & \multirow{2}{*}{$\begin{array}{l}\text { Ordem do } \\
\text { controle } \\
\text { Control } \\
\text { number }\end{array}$} & \multirow{2}{*}{$\begin{array}{l}\text { Produção } \\
\text { observada } \\
\text { Observed } \\
\text { yield }\end{array}$} & \multicolumn{5}{|c|}{$\begin{array}{l}\text { Produção prevista } \\
\text { Predicted yield }\end{array}$} \\
\hline & & & $\begin{array}{c}\text { Gama Incom. } \\
\text { Incomplete gamma }\end{array}$ & $\begin{array}{l}\text { Monofásica } \\
\text { Monophasic }\end{array}$ & $\begin{array}{l}\text { Difásica } \\
\text { Diphasic }\end{array}$ & $\begin{array}{c}\text { Difásica } \\
\text { Quad. Logarith. }\end{array}$ & $\begin{array}{c}\text { Reg. Múlt. } \\
\text { Multi. Regres. }\end{array}$ \\
\hline 22 & 1 & 24,44 & 24,5550 & 24,4361 & 24,5116 & 24,4361 & 24,4419 \\
\hline 52 & 2 & 24,89 & 24,6628 & 24,8905 & 24,7128 & 24,8905 & 24,8819 \\
\hline 82 & 3 & 23,86 & 23,7784 & 23,8624 & 23,9434 & 23,8624 & 23,8417 \\
\hline 112 & 4 & 22,47 & 22,5951 & 22,4707 & 22,6244 & 22,4707 & 22,5504 \\
\hline 142 & 5 & 21,27 & 21,3098 & 21,2733 & 21,1859 & 21,2733 & 21,2226 \\
\hline 172 & 6 & 19,96 & 20,0050 & 19,9581 & 19,8399 & 19,9581 & 19,9193 \\
\hline 202 & 7 & 18,64 & 18,7216 & 18,6375 & 18,6163 & 18,6375 & 18,6610 \\
\hline 232 & 8 & 17,45 & 17,4811 & 17,4527 & 17,4737 & 17,4527 & 17,4546 \\
\hline 262 & 9 & 16,26 & 16,2949 & 16,2571 & 16,3669 & 16,2571 & 16,3018 \\
\hline 292 & 10 & 15,29 & 15,1691 & 15,2942 & 15,2688 & 15,2942 & 15,2023 \\
\hline 322 & 11 & 14,07 & 14,1058 & 14,0675 & 14,1710 & 14,0675 & 14,1548 \\
\hline 352 & 12 & 13,19 & 13,1056 & 13,1944 & 13,0772 & 13,1944 & 13,1578 \\
\hline \multirow{2}{*}{\multicolumn{3}{|c|}{$\begin{array}{l}\mathrm{R}^{2} \text { ajustado }{ }^{1} \\
\text { Adjusted } R^{2} \\
\text { Auto correlação } 1^{\mathrm{a}} \text { ordem }\end{array}$}} & 0,999967 & 0,999676 & 0,999950 & 0,999974 & 0,999756 \\
\hline & & & $-0,1398 \mathrm{~ns}$ & $0,0406 \mathrm{~ns}$ & $0,2487 \mathrm{~ns}$ & $-0,0993 \mathrm{~ns}$ & $-0,6097 \mathrm{~ns}$ \\
\hline \multicolumn{3}{|c|}{ Desvio-padrão residual ${ }^{3}$} & 0,1185 & 0,3707 & 0,1455 & 0,1045 & 0,1700 \\
\hline \multicolumn{3}{|c|}{ Teste de Durbin-Watson ${ }^{4}$} & $2,1053 \mathrm{~ns}$ & $1,4920 \mathrm{nc}^{5}$ & $2,3445 \mathrm{~ns}$ & $2,1372 \mathrm{~ns}$ & $3,1835 \mathrm{~ns}$ \\
\hline
\end{tabular}

${ }^{1} \mathrm{R}^{2}$ ajustado ${ }^{\circledR} \mathrm{RA}{ }^{2}=1-((\mathrm{N}-1) /(\mathrm{N}-\mathrm{P}))\left(1-\mathrm{R}^{2}\right)$, em que $\mathrm{N}=\mathrm{n}^{\circ}$ de obs; $\mathrm{P}=\mathrm{n}^{\circ}$ de parâmetros $\left(\right.$ Adjusted $R^{2} \circledast R A^{2}=1-((\mathrm{N}-1) /(\mathrm{N}-\mathrm{P}))\left(1-R^{2}\right)$, where $N=$ number of observations $P=$ number of parameters $)$.

2 Distribuição da autocorrelação de resíduo de primeira ordem (Error autocorrelation of first order distribution).

3 Raiz quadrada do quadrado médio do resíduo da regressão (Square root of the regression error mean square).

4 Conforme Giron-Pineda (1987) (According Giron-Pineda [1987]).

5 não conclusivo (inconclusive).

R. Bras. Zootec., v.31, n.4, p.1689-1694, 2002 


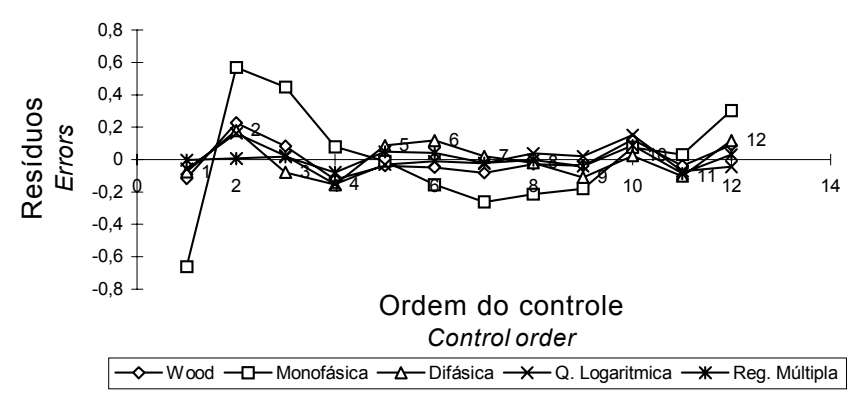

Figura 1 - Distribuição dos resíduos das diferentes curvas de lactação.

Figure 1 - Distribution of residuals for different lactation curves.

Segundo Grossmann \& Koops (1988), as funções das estimativas dos parâmetros apresentadas na Tabela 2 são úteis para descrever a curva de lactação. A produção inicial para os modelos gamma incompleto, quadrático-logarítmico e de regressão múltipla é estimada em zero, o pico de produção foi semelhante para esses três modelos $(24,8 ; 24,9$ e $25,1 \mathrm{~kg}$, respectivamente), os valores do tempo até o pico, tempo em dias em que ocorre a maior produção, foram próximos $(36,7 ; 38,4$ e 38,6 dias, respectivamente), não sendo estimada a duração da fase.

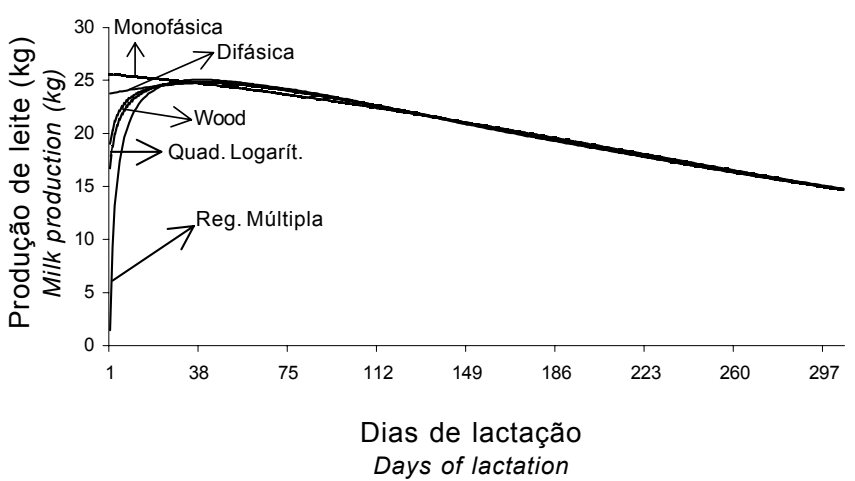

Figura 2 - Produção de leite em função dos dias de controle das diferentes curvas estudadas.

Figure 2 - Milk yield according to the control days of the different studied lactation curves.

Pela função monofásica, a produção inicial foi $25,6 \mathrm{~kg}$, o pico, $26,5 \mathrm{~kg}$ de leite e o tempo até o pico, de 94,5 dias, sugerindo que a função é inadequada, visto que o tempo até o pico é quase três vezes superior ao dos demais modelos; a duração da fase foi 990 dias, valor muito superior ao de uma fase normal de 305 dias, mas, Grossmann \& Koops (1988) também encontraram o valor $(632,9)$ bem acima de 305 dias para essa função, ao passo que a produção em 305 dias $(6292,3 \mathrm{~kg})$ foi superior à obtida usando-se os modelos anteriores.

Tabela 2 - Funções das estimativas dos parâmetros obtidas por meio dos diferentes modelos

Table 2 - Function of estimates of parameters obtained by different models

\begin{tabular}{|c|c|c|c|c|c|c|}
\hline $\begin{array}{l}\text { Modelos } \\
\text { Models }\end{array}$ & $\begin{array}{l}\text { Fase } \\
\text { Phase }\end{array}$ & $\begin{array}{l}\text { Produção } \\
\text { inicial }(\mathrm{kg})^{1} \\
\text { Initialyield }\end{array}$ & $\begin{array}{c}\text { Pico de } \\
\text { produção }(\mathrm{kg})^{2} \\
\text { Peakyield }\end{array}$ & $\begin{array}{c}\text { Produção em } \\
305 \text { dias }(\mathrm{kg})^{3} \\
\text { 305-dayyield }\end{array}$ & $\begin{array}{c}\text { Tempo até } \\
\text { o pico (dias) })^{4} \\
\text { Time of peak }\end{array}$ & $\begin{array}{c}\text { Duração da } \\
\text { fase (dias) })^{5} \\
\text { Duration of phase }\end{array}$ \\
\hline $\begin{array}{l}\text { Gama incompleta } \\
\text { Incomplete gamma }\end{array}$ & & 0 & 24,8 & 6245,7 & 36,70 & - \\
\hline $\begin{array}{l}\text { Monofásica } \\
\text { Monophasic }\end{array}$ & 1 & 25,6 & 26,5 & 6292,3 & 94,5 & 990,1 \\
\hline $\begin{array}{l}\text { Difásica } \\
\text { Diphasic }\end{array}$ & $\begin{array}{c}1 \\
2 \\
\text { Total }\end{array}$ & $\begin{array}{r}5,1 \\
18,6 \\
23,7\end{array}$ & $\begin{array}{r}5,7 \\
19,4\end{array}$ & $\begin{array}{r}785,2 \\
5481,6 \\
6266,8\end{array}$ & $\begin{array}{l}34,3 \\
80,4\end{array}$ & $\begin{array}{l}212,8 \\
833,3\end{array}$ \\
\hline $\begin{array}{l}\text { Quad. logar. } \\
\text { Quadratic logarithmic } \\
\text { Reg. múltipla } \\
\text { Multiple regression }\end{array}$ & & - & 24,88 & 6231,1 & 38,4 & - \\
\hline
\end{tabular}

${ }^{1}$ Estimado pelo modelo, com $\mathrm{t}=0$ (Estimated by model for $t=0$ ).

$2 \mathrm{a}_{\mathrm{i}}\left(b_{i} / c_{i}\right)^{2} e^{-b_{i}}$ para modelo gama, aibi para multifásicos, ai+bit+cit2+di In(t) para modelo quadrático logarítmico e estimado graficamente para modelo de regressão múltipla $\left(\mathrm{a}_{\mathrm{i}}\left(b_{i} / c_{i}\right)^{2} e^{-b_{i}}\right.$ for the gamma model, aibi for the multiphasic models, ai+bit+cit2+di In $(t)$ for the quadratic logarithmic model and estimate graphically for the multiple regression model).

3 Utilização do modelo, sendo o somatório de 0 a 305 dias (Model utilization, being $S$ from 0 to 305 days).

${ }^{4} \mathrm{bi} / \mathrm{ci}$ para modelo gama, ci para multifásicos, $\left(-\mathrm{b}_{\mathrm{i}} \pm \sqrt{\mathrm{b}_{\mathrm{i}}{ }^{2}-8 \mathrm{~b}_{\mathrm{i}} \mathrm{c}_{\mathrm{i}} \mathrm{d}_{\mathrm{i}}}\right) / 4 \mathrm{c}_{\mathrm{i}}$ para modelo quadrático logarítmico e estimado graficamente para modelo de regressão múltipla (Bi/ci for the gamma model, ci for the multiphasic models, $\left(-\mathrm{b}_{\mathrm{i}} \pm \sqrt{\mathrm{b}_{\mathrm{i}}{ }^{2}-8 \mathrm{~b}_{\mathrm{i}} \mathrm{c}_{\mathrm{i}} \mathrm{d}_{\mathrm{i}}}\right) / 4 \mathrm{c}_{\mathrm{i}}$ for the quadratic logarithmic model and estimate graphically for the multiple regression model).

$52 \mathrm{bi}^{-1}(\mathrm{~d})$.

R. Bras. Zootec., v.31, n.4, p.1689-1694, 2002 
Pela Figura 3 pode ser visualizada a forma da curva que melhor estimou a produção de leite, mostrando a natureza difásica da lactação, com uma primeira fase, que é responsável pelo pico de produção, à qual se sobrepõe uma segunda fase que define a persistência da lactação. Esse resultado é semelhante aos obtidos por Grossmann \& Koops (1988), De Boer et al. (1989) e Sherchand et al. (1995), todos nos Estados Unidos, e Vargas et al. (2000), na Costa Rica.

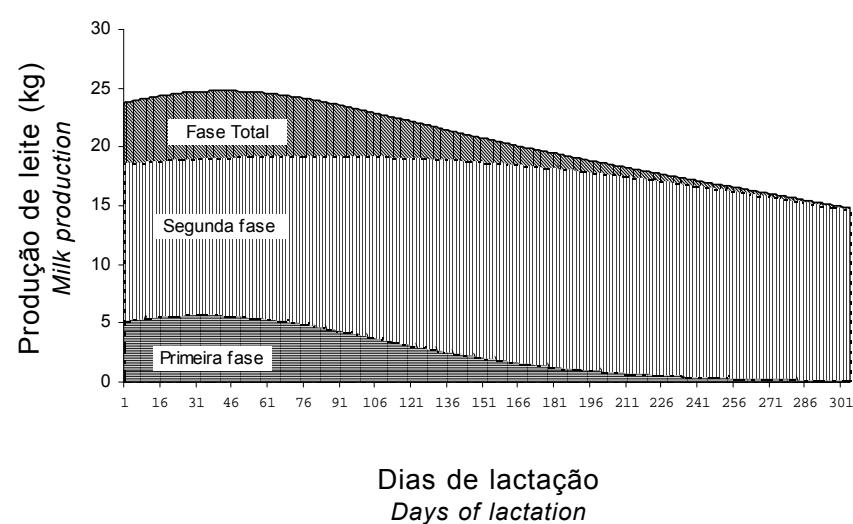

Figura 3 - Produção estimada de leite $(\mathrm{kg})$ pela curva difásica em função dos dias de lactação

Figure 3 - Estimated milk yield $(\mathrm{kg})$ by diphasic curve according to the lactation days
No caso do modelo difásico, aproximadamente $21,7 \%$ da produção inicial é explicada pela primeira fase da lactação $(5,1 \mathrm{~kg})$ e $78,3 \%$ pela segunda fase $(18,6 \mathrm{~kg})$, perfazendo uma produção inicial total de $23,7 \mathrm{~kg}$. Para a primeira fase, a produção inicial correspondeu à cerca de $89,5 \%$ do pico de produção para aquela fase $(5,7 \mathrm{~kg})$, inferindo-se que a produção inicial contribuiu razoavelmente para o pico de produção, semelhantemente à segunda fase, na qual a produção inicial foi $95,9 \%$ do pico de produção $(19,4 \mathrm{~kg})$, ou seja, a produção inicial foi praticamente o pico de produção. Apenas $12,5 \%$ da produção total aos 305 dias $(6266,8 \mathrm{~kg})$ foi atingida durante a primeira fase, sendo o restante produzido na segunda fase. O tempo até o pico foi de 34,3 dias para a primeira fase e 80,4 dias para a segunda. A duração da primeira fase (212,8 dias) foi menor que aquela obtida para a segunda fase (833,3 dias), inferindo-se que enquanto a segunda fase da lactação ocorreu durante todo o período de 305 dias, a primeira fase da lactação ocorreu apenas durante os dois terços iniciais da lactação (Figura 3).

A função difásica, com resíduos menores, mais simétricos e menos correlacionados que as demais, é suficiente para descrever a produção de leite para esses dados.

Os coeficientes das equações para o modelo difásico são apresentados na Tabela 3 , sendo, então,

Tabela 3 - Coeficientes do modelo difásico ${ }^{1}$ para as diferentes classes de ordem de parição e idade ao parto, de acordo com os níveis de produção

Table 3 - Coefficients of the difasic model for the different classes of parity order and calving age, according to the management levels

\begin{tabular}{lcrrrrrr}
\hline $\begin{array}{l}\text { Ordem de parição } \\
\text { Parity order }\end{array}$ & $\begin{array}{c}\text { Idade ao parto (meses) } \\
\text { Calving age (months) }\end{array}$ & \multicolumn{1}{c}{$\hat{A}_{1}$} & $\hat{B}_{1}$ & $\hat{C}_{1}$ & $\hat{A}_{2}$ & $\hat{B}_{2}$ & $\hat{C}_{2}$ \\
\hline & & & \multicolumn{4}{c}{ Nível baixo de produção (Production low level) } \\
1 & $<30$ & 557,743 & 0,00864 & 41,195 & 7534,947 & 0,00188 & 92,796 \\
1 & $30-42$ & 1660,477 & 0,00557 & $-2,622$ & 5260,672 & 0,00225 & 191,619 \\
2 & $<42$ & 820,996 & 0,10381 & 944,832 & 14678,470 & 0,00182 & $-207,481$ \\
2 & $42-60$ & 285,672 & 0,01136 & 36,123 & 10375,530 & 0,00193 & $-32,270$ \\
3 & $<60$ & 722,584 & 0,00923 & 27,963 & 6748,720 & 0,00258 & 113,412 \\
3 & $60-72$ & 421,900 & 0,01013 & 37,765 & 10865,359 & 0,00195 & $-40,987$ \\
3 & $>72$ & 392,244 & 0,01116 & 38,600 & 9186,299 & 0,00222 & 6,284 \\
& & & & Nível alto de produção (Production high level) & \\
1 & $<30$ & 1862,066 & 0,00706 & 34,926 & 4697,147 & 0,00345 & 275,746 \\
1 & $30-42$ & 2073,043 & 0,00570 & 30,518 & 5811,236 & 0,00247 & 253,657 \\
2 & $<42$ & 607,451 & 0,01017 & 39,595 & 8231,413 & 0,00261 & 82,807 \\
2 & $42-60$ & 791,142 & 0,01130 & 29,264 & 6218,994 & 0,00350 & 139,335 \\
3 & $<60$ & 757,330 & 0,01117 & 28,383 & 6902,571 & 0,00330 & 123,182 \\
3 & $60-72$ & 922,061 & 0,00882 & 32,210 & 9657,179 & 0,00229 & 83,031 \\
3 & $>72$ & 8388,015 & 0,00358 & 22,235 & 11490,523 & 0,00571 & 649,651 \\
\hline
\end{tabular}

${ }^{1}$ Modelo difásico: $\mathrm{Y}=\mathrm{A} 1^{*} \mathrm{~B} 1{ }^{*}\left(1-\tanh 2\left(\mathrm{~B} 1{ }^{*}(\mathrm{t}-\mathrm{C} 1)\right)\right)+\mathrm{A} 2{ }^{*} \mathrm{~B} 2{ }^{*}\left(1-\tanh 2\left(\mathrm{~B} 2{ }^{*}(\mathrm{t}-\mathrm{C} 2)\right)\right)$

Diphasic model: $Y=A 1^{*} B 1^{*}(1-\tanh 2(B 1 *(t-C 1)))+A 2{ }^{*} B 2 *(1-\tanh 2(B 2 *(t-C 2)))$.

R. Bras. Zootec., v.31, n.4, p.1689-1694, 2002 
possível, para cada nível de produção e cada classe de ordem de parição e idade ao parto, estimar a produção de leite em 305 dias em rebanhos da raça Holandesa a partir do modelo difásico.

\section{Conclusões}

Com base nos resultados, é possível afirmar que a função difásica ajusta-se adequadamente a dados de produção de leite, podendo ser usada para estimar produções de leite em trabalhos de seleção.

\section{Literatura Citada}

ALI, T.E.; SCHAEFFER, L.R. Accounting for covariances among test day milk yields in dairy cows. Canadian Journal of Animal Science, v.67, p.637-644, 1987.

BRODY, S.; TURNER, C.W.; RAGSDALE, A.C. The rate of decline of milk secretion with the advance of the period lactation. Journal of Genetic Physiology, n.5, p.441, 1923.

BROWN, J.E.; FITZHUGH Jr., H.A.; CARTWRIGHT, T.C. A comparison of nonlinear models for describing weight-age relationships in cattle. Animal Production, v.42, n.2, p.810818. 1976

COBBY, J.M.; LE DU, Y.L.P. An fitting curves to lactation data. Animal Production, v.26, n.2, p.127-133, 1978.

DE BOER, J.A., WELLER, J.I., GIPSON, T.A.; GROSSMAN, M. 1989. Multiphasic analysis of milk and fat yield curves of Israeli Holstein. Journal of Dairy Science, v.72, n.7, p.2143-2152.
GIRON-PINEDA, A.M. Mathematical functions applied to dairy cows lactation curves. Florida: University of Florida, 1987. 243p.

GROSSMAN, M.; KOOPS, W.J. Multiphasic analysis of lactation curves in dairy cattle. Journal of Dairy Science, v.71, n.5, p.1598-1608, 1988.

HOFFMANN, R.; VIEIRA, S.O. O teste de Durbin-Watson. In Análise de regressão; uma introdução à econometria. 2ed. São Paulo: Hucitec, 1987. p.251-256.

MALHOTRA, P.K.; SING, P.R.; SING, N.R. Estimating lactation curve in Karan-Swiss cattle. Indian Journal of Animal Science, v.30, n.10, p.799-804, 1980.

SHERCHAND, L.; McNEW, R.W.; KELLOG, D.W.; JOHNSON, Z.B. Selection of a mathematical model to generate lactation curves using daily milk yields of Holstein cows. Journal of Dairy Science, v.78, n.8, p.2507-2513, 1995.

VARGAS, B.; KOPS, W.J.; HERRERO, M. et al. Modeling extended lactations of dairy cows. Journal of Dairy Science, v.83, n.4, p.1371-1380, 2000.

WOOD, P.D.P. Algebric of the lactation curve in cattle. Nature, n.216, p.164-165, 1967.

WILMINK, J.B.M. Efficiency of selection for different cumulative milk, fat and protein yields in first lactation. Livestock Production Science, v.17, p.211, 1987.

YADAV, M.C.; KATPATAL, B.C.; KAVSHIK, S.N. Components of gamma type function of a lactation curve, and factors affecting them in Hariana and its Friesian crosse. Indian Journal of Animal Science, v.49, n.9, p.502-505, 1977. 